\title{
生态阈值：概念、方法与研究展望
}

\author{
唐海萍 ${ }^{*}$ 陈 姣 薛海丽 \\ 北京师范大学地表过程与资源生态国家重点实验室, 北京师范大学资源学院, 北京 100875
}

摘 要 生态阈值概念是20世纪70年代提出的, 主要指生态系统的几个稳态之间突然改变的点或区域。在阐明生态系统结 构与功能的关系、构建区域可持续发展范式以及服务于生态系统管理和生态红线的划定中, 生态阈值的检测和量化有着重要 的理论和实践意义。该文首先梳理了前人关于生态阈值的概念、类型的一些提法, 从预警研究角度提出可以从两个层次理解 生态阈值概念: 生态阈值点是系统从量变到质变的转折点, 类似于红色界限; 而生态阈值带可以理解为量变过程中不同稳态 之间的转换区域, 类似于黄色与橙色预警边界带。黄色生态阈值表示生态系统可通过自身的调节能力重新达到稳定状态; 橙 色生态阈值表示需要排除干扰因子使得生态系统重新达到平衡; 而红色生态阈值为关键阈值点, 超过此阈值, 生态系统将发 生不可逆的退化甚至崩溃。该文还总结了目前确定生态阈值的主要方法, 主要是基于野外观测数据的统计分析与模型模拟方 法。最后, 基于生态系统服务、生物多样性保护与生态系统管理等几个当今生态学热点研究领域, 简单总结归纳了生态阈值 的研究现状, 并提出生态阈值未来的 3 个研究难点和方向: 1)开展针对生态阈值检测和量化的研究; 2)关注生态阈值的尺度效 应并加强野外观测; 3)发挥生态阈值的预警作用, 指导“生态红线”的划定和生态系统管理。

关键词 生态阈值, 稳态转换, 恢复力, 生态系统管理, 生态系统服务, 生态红线

引用格式: 唐海萍, 陈姣, 薛海丽 (2015). 生态阈值: 概念、方法与研究展望. 植物生态学报, 39, 932-940. doi: 10.17521/cjpe.2015.0090

\section{Ecological thresholds: Concept, methods and research outlooks}

TANG Hai-Ping*, CHEN Jiao, and XUE Hai-Li

State Key Laboratory of Earth Surface Processes \& Resource Ecology, Beijing Normal University, College of Resources Science \& Technology, Beijing Normal University, Beijing 100875, China

\section{Abstract}

The concept of ecological thresholds was raised in the 1970s. However, it was subsequently given different definitions and interpretations depending on research fields or disciplines. For most scientists, ecological thresholds refer to the points or zones that link abrupt changes between alternative stable states of an ecosystem. The measurement and quantification of ecological thresholds have great theoretical and practical significance in ecological research for clarifying the structure and function of ecosystems, for planning sustainable development modes, and for delimiting ecological red lines in managing the ecosystems of a region. By reviewing the existing concepts and classifications of ecological thresholds, we propose a new concept and definition at two different levels: the ecological threshold points, i.e. the turning points of quantitative changes to qualitative changes, which can be considered as ecological red lines; the ecological threshold zones, i.e. the regime shifts of the quantitative changes among different stable states, which can be considered as the yellow and/or orange warning boundaries of the gradual ecological changes. The yellow thresholds mean that an ecosystem can return to a stable state by its self-adjustment, the orange thresholds indicate that the ecosystem will stay in the equilibrium state after interference factors being removed, whereas the red thresholds, as the critical threshold points, indicate that the ecosystem will undergo irreversible degradation or even collapse beyond those points. We also summarizes two types of popular methods in determining ecological thresholds: statistical analysis and modeling based on data of field observations. The applications of ecological thresholds in ecosystem service, biodiversity conservation and ecosystem management research are also reviewed. Future research on ecological thresholds should focus on the following aspects: (1) methodological development for measurement and quantification of ecological thresholds; (2) emphasizing the scaling effect of ecological thresholds and establishment of national-scale observation system and network; and (3) implementation of ecological thresholds as early warning tools in ecosystem management

收稿日期Received: 2015-02-03 接受日期Accepted: 2015-07-23

* 通讯作者Author for correspondence (E-mail: tanghp@bnu.edu.cn) 
and delimiting ecological red lines.

Key words ecological thresholds, regime shifts, resilience, ecological management, ecosystem service, ecological red line

Citation: Tang HP, Chen J, Xue HL (2015). Ecological thresholds: Concept, methods and research outlooks. Chinese Journal of Plant Ecology, 39, 932-940. doi: 10.17521/cjpe. 2015.0090

Future Earth (2013)计划的研究问题之一是探究 地球生命承载力极限与临界点, 对全球环境变化对 人类的食物、水、健康和能源等需求进行早期预警。 在预警研究中, 阈值成为一个关键因子。阈值用于 生态系统的研究, 于是衍生出生态阈值概念。生态 阈值是自然科学、地球科学、社会科学近年来的重 要研究方向, 是可持续性科学研究的核心问题之一 (Kates et al., 2001), 不仅对了解生态系统结构功能 具有十分重要的作用, 而且对生态系统管理有潜在 的应用价值(Huggett, 2005; Groffman et al., 2006)。 由于生态系统的复杂性, 结构和功能特性在不同的 研究尺度表现不同, 这种生态阈值的非线性变化体 现在生态系统的多稳态转换之中。生态阈值最初应 用于半干旱区放牧生态系统与湖泊生态系统 (Laycock, 1991; Perrings \& Walker, 1997), 现在应用 领域扩大到更多生态系统类型和不同研究尺度 (Suding et al., 2004; Suding \& Hobbs, 2009; Swift \& Hannon, 2010), 以及与可持续科学相关的社会-经 济-生态复合系统(Armitage et al., 2012)。该文从生 态阈值的概念、类型、研究方法及相关应用等几方
面梳理已有国内外文献，提出我们对生态阈值概念 的理解以及对生态阈值的研究展望, 特别指出生态 阈值研究可以作为国家生态红线划定中的一个重要 科学基础, 发挥生态阈值的分级预警作用可为政策 制定和决策提供服务。

\section{1 生态阈值的概念和类型}

生态阈值的概念来自Holling (1973)提出的生态 系统具有多个稳定状态的理论。May (1973)针对生 态系统的多个稳定状态的实验观测提出了阈值理论 模型(theoretical models), 促进了生态阈值研究的发 展。从 20 世纪 90 年代开始, 生态阈值的研究论文数 量呈指数增长, 我们共搜集到 4021 篇相关文献; 国 内从2002年开始开展生态阈值的相关研究(图1)。生 态阈值概念的发展因研究对象和研究目标的不同而 有不同的理解(表1)。目前对生态阈值的概念描述尚 不统一。非平衡系统概念(DeAngelis \& Waterhouse, 1987)的提出是阈值概念发展的重要理论依据。本文 梳理了在生态阈值概念表述中经常被提到的一些科 学术语及定义(表2)。

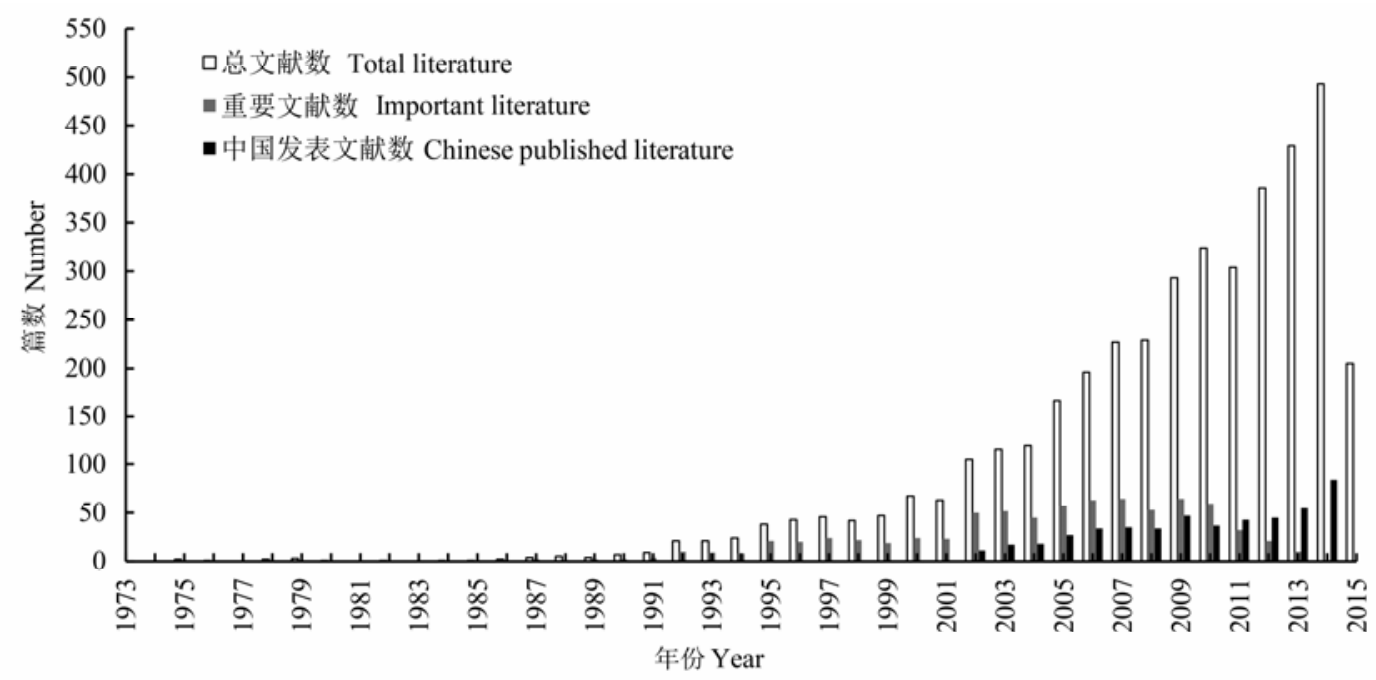

图1 1973年1月-2015年6月发表的有关生态阈值的论文(来自Web of Science数据库检索)。图中重要文献指引用频次 $>30$ 次的 文献。

Fig. 1 Literature on ecological thresholds published during 1973.1-2015.6 (Resource: Web of Science). Important literature are those with citation frequency of more than 30 times. 
表1 生态阈值概念

Table 1 The concepts of ecological thresholds

\begin{tabular}{|c|c|}
\hline 概念 Concept & 文献 Reference \\
\hline $\begin{array}{l}\text { 系统两个稳定状态之间的断点代表了生态系统阈值 } \\
\text { The breakpoints between alternative stable states }\end{array}$ & May, 1977 \\
\hline $\begin{array}{l}\text { 阈值是两个不同的生态状态之间的时空边界; 牧区管理者没有实质干预的在实践时间尺度上不可逆的初始跨越边界 } \\
\text { The boundaries in space and time between two different ecological states, initial shift across the boundary is not reversible on a } \\
\text { practical time scale without substantial intervention by the range manager }\end{array}$ & Friedel, 1991 \\
\hline $\begin{array}{l}\text { 生态阈值是生态的不连续性, 暗示系统从一个稳态跃入另一个稳态时独立变量的关键值 } \\
\text { The critical values of the independent variable around which the system flips from one stable state to another. }\end{array}$ & Muradian, 2001 \\
\hline $\begin{array}{l}\text { 阈值是环境条件的小变化产生(系统功能等)实质性改进的一些区域 } \\
\text { The zones at which small changes in environmental conditions produce substantial improvements }\end{array}$ & Bestelmeyer et al., 2004 \\
\hline $\begin{array}{l}\text { 定义生态阈值为系统中当跨越两个可选状态时引起系统“快速移动”到一个不同的状态时的分歧点 } \\
\text { A bifurcation point between alternate states which when passed causes a system to “flip” to a different state }\end{array}$ & Meyers \& Walker, 2003 \\
\hline $\begin{array}{l}\text { 生态阈值代表了生态过程或参数发生突变的一个点, 此突变点响应于一个驱动力的相对较小的变化 } \\
\text { The point at which an ecological process or parameter changes abruptly in response to relatively small change }\end{array}$ & Larsen \& Alp, 2015 \\
\hline
\end{tabular}

表2 几个与生态阈值有关的术语

Table 2 Several terms related to ecological thresholds

\begin{tabular}{|c|c|c|}
\hline 术语 Term & 定义 Definition & 文献 Reference \\
\hline $\begin{array}{l}\text { 弹性或恢复力 } \\
\text { Resilience }\end{array}$ & $\begin{array}{l}\text { 系统持久性的度量, 以及吸纳变化和干扰后维持同样的种群或状态变量之间关系的能力; } \\
\text { A measure of the persistence of system and their ability to absorb change and disturbance and to still } \\
\text { maintain the same relationship between populations or state variables }\end{array}$ & Holling, 1973 \\
\hline $\begin{array}{l}\text { 非线性变化 } \\
\text { Non-linear responses }\end{array}$ & $\begin{array}{l}\text { 非线性变化是阈值的重要原理 } \\
\text { Ecosystem reflects different types of nonlinearities }\end{array}$ & Rial et al., 2004 \\
\hline $\begin{array}{l}\text { 触发因子 } \\
\text { Triggers }\end{array}$ & $\begin{array}{l}\text { 触发因子经常引起生态系统格局或空间结构发生初始变化 } \\
\text { Triggers often initiate changes in the pattern or spatial structure of ecosystem }\end{array}$ & Bowker et al., 2014 \\
\hline $\begin{array}{l}\text { 稳态转换 } \\
\text { Regime shift }\end{array}$ & $\begin{array}{l}\text { 生态系统稳态转换意味着生态系统服务的转变以及随后对人类社会的影响 } \\
\text { Regime shifts imply shifts in ecosystem services and consequent impacts on human societies }\end{array}$ & Folke et al., 2004 \\
\hline $\begin{array}{l}\text { 临界点 } \\
\text { Tipping points }\end{array}$ & $\begin{array}{l}\text { (1)复杂系统的临界点可能隐含非必要崩溃的风险, 同时也是积极改变的机会 } \\
\text { Tipping points in complex systems may imply risks of unwanted collapse, but also opportunities } \\
\text { for positive change }\end{array}$ & Scheffer et al., 2012 \\
\hline & $\begin{array}{l}\text { (2)指示阈值出现拐点或者迫在眉睫就要出现 } \\
\text { Indicate that thresholds crossing has occurred or is imminent }\end{array}$ & Bowker et al., 2014 \\
\hline $\begin{array}{l}\text { 多稳态 } \\
\text { alternative stable states } \\
\text { (multiple stable states) }\end{array}$ & $\begin{array}{l}\text { 描述这种现象, 即系统能展示从一种稳态到另一种稳态发生的重大变化 } \\
\text { describe the phenomenon whereby systems can exhibit a big change from one kind of regime to an- } \\
\text { other }\end{array}$ & Walker \& Meyers, 2004 \\
\hline $\begin{array}{l}\text { 断点 } \\
\text { Breakpoint }\end{array}$ & $\begin{array}{l}\text { 多稳态之间的过渡点 } \\
\text { transition point to alternative stable states }\end{array}$ & May, 1977 \\
\hline 不连续性 & 通过这些断点或不连续性识别生态阈值和描述多稳态之间的转换过程 & Muradian, 2001 \\
\hline Discontinuities & $\begin{array}{l}\text { Identifying ecological thresholds and describing processes of transition to alternative stable states once } \\
\text { these breakpoints or discontinuities }\end{array}$ & \\
\hline
\end{tabular}

Bestelmeyer (2006)基于牧场预防管理和恢复认 为生态阈值有两种分类方法: 其一, 分为格局阈值 (pattern thresholds)、过程阈值(process thresholds)和 退化阈值(degradation thresholds) 3种类型; 其二, 分为预防阈值 (preventive thresholds) 和恢复阈值 (restoration thresholds)。预防管理必须关注对易使系 统受到确定性或事件驱动的格局变化的调控, 当管 理失败时, 格局阈值无法及时指示生态系统的退化, 而退化阈值就成为重要的指标。相反, 退化草地的 恢复需要同时确定格局阈值、过程阈值和退化阈值。 Groffman等(2006)提出阈值概念应用于生态学研究 领域, 即生态阈值, 它主要有3种类型：生态系统状 态的转换、临界负荷和外部因子阈值。生态系统状
态转换时, 系统运行的驱动力的一个微小的变化就 会引起生态系统条件的明显改变, 这就是生态系统 状态的转换; 临界负荷即生态系统状态和(或)特定 生态系统功能改变前, 生态系统可安全吸纳的污染 物数量; 外部因子阈值指大尺度上一个变量的变化 改变了小尺度上驱动力与响应之间的关系。Martin 等(2009)根据自然资源管理与保护的实际需要, 基 于模型中主观的管理目标和潜在的管理行为, 以及 客观的系统模型和系统状态估计，从保护决策的需 要把阈值分为生态阈值(ecological thresholds)、利用 阈值(utility thresholds)、决定阈值(decision thresholds) 3类。生态阈值是生态系统状态变量的值, 其微 小的改变会造成系统动态的实质性变化; 利用阈值 
是基于人类价值决定的管理目标的组分, 是状态或 性能变量的值, 其微小的变化能导致管理价值结果 的实质改变; 决定阈值指生态系统状态变量的值, 其微小的变化能迅速促使管理者改变具体管理行动 以达到既定的管理目标。

从表1可以看出, 前人对生态阈值的理解主要 是生态阈值点(ecological thresholds point)和生态阈 值带(ecological thresholds zone)。Huggett (2005)提 出, 理论上自然系统中有两种主要的阈值类型: 点 和带。我们认为可以用哲学上量变和质变的关系来 理解点和带。生态阈值点强调系统两个稳态之间的 突然转变是生态系统从量变到质变的转折点, 此时 驱动因子的微小变化都可能导致生态系统的不可逆 变化; 而生态阈值带表明系统的两个稳态之间的跃 升或下降需要经历一个过渡或者重合区域，可以理 解为量变过程中不同稳定状态之间的转换区域, 此 时驱动因子的变化可以加剧生态系统稳态之间的转 换, 也可以减缓或者逆袭这种转换, 在阈值带, 生 态系统状态的任何变化都是可逆的。

因此，我们应当关注生态阈值带中能够体现生 态系统重大突变的关键國值, 例如在群落生态学研 究中, 关键阈值可以理解为演替不同阶段之间转换 的阈值。而在恢复生态学研究中, 关键國值体现在 生态系统结构与功能的阶段性稳定状态。我们可以 利用野外观察或用模型模拟出的生态阈值点和带的 量变积累到质变的这一特性, 为生态系统管理提供 预警服务。根据生态阈值在管理中的应用, 可以将 生态阈值分为不同等级: 红色生态阈值、橙色生态 阈值和黄色生态阈值。黄色生态阈值表示生态系统 可以通过自身的调节能力, 也即系统的持久性 (persistance), 重新达到稳定状态; 橙色生态阈值表 示需要移除干扰因子, 利用系统的弹性或者说恢复 力(resilience)重新回到平衡状态; 而红色生态阈值 为关键國值点, 超过此阈值, 生态系统将发生不可 逆的转换甚至系统崩溃。

\section{2 生态阈值的研究方法}

弹性联盟(The Resilience Alliance)在线数据库 列出了47种生态阈值的不同案例(Huggett, 2005)。生 态阈值的检测(detect)和量化是生态学研究的重大 挑战, 与生态系统功能及生态系统管理密切相关 (Huggett, 2005; 赵慧霞等, 2007; Ficetola \& Denoël,
2009)。非线性变化是生态阈值的重要原理(Rial et al., 2004), 生态阈值的确定是通过生态系统的动态 变化过程研究, 找出非线性变化拐点, 从而确定生 态阈值。

早期的阈值确定主要是通过野外观察, 如 Friedel (1991)提出的3种阈值例子, 在干旱半干旱牧 区把草地和林地分开的阈值, 稳定和退化的土壤之 间的阈值以及由适口性好的多年生植物种变为适口 性好但短命的植物种。目前阈值的定量研究还处于 萌芽阶段(Scheffer et al., 2009), 常用的生态阈值的 确定方法主要有统计分析(应用统计模型, Meta分 析)和模型模拟(过程模型、系统动力学模型、概念 模型等)两种方法, 而这两种方法都是基于大量野 外数据获取(野外观测、遥感监测和大尺度的观测网 络)的。几种方法之间均存在交叉, 不可能绝对区分 开, 要以问题导向为依据来确定生态阈值, 在此过 程中选择适合的方法。

\section{1 概念模型}

概念模型可以揭示生态系统的动态过程, 通过 概念模型的建立和实验数据获取, 结合定量方法即 可确定生态阈值。目前在生态阈值研究中广泛应用 的为状态与过程模型(state and transition models)可 用于描述和理解牧场生态系统不同结构与功能的状 态，以及改变这些状态的过程(Bestelmeyer et al., 2004), 基于状态过程模型发展的一些概念模型, 如 Fire-Vegetation模型等也被广泛用于对生态阈值的 研究(Twidwell et al., 2013)。数据获取主要为野外观 测、遥感监测和大尺度的观测网络, 通过野外观测 能够实现生态系统各类要素的实时观测, 准确反映 生态系统对环境变化响应的规律, 是目前生态阈值 研究的基础手段(Wang et al., 2014)。针对生态阈值 确定的野外观测主要包括控制实验、样带设置、不 同演替阶段生态系统结构功能观测等方法。控制实 验是研究生态阈值的重要手段, 不仅可以研究特定 环境因子变化对生态系统产生的影响, 而且可以模 拟全球变化不同情景下生态系统的结构变化阈值 (Bai et al., 2010), 但控制试验的研究结果需要结合 野外观测, 才能真实地反映自然条件下生态系统的 状态和阈值。样带设置可以通过对景观尺度上环境 因子变化的观测, 得出随自然因子梯度变化所产生 的生态阈值, 研究结果可以较为准确地应用在管理 中(Sasaki et al., 2008), 且随着研究手段的提高, 结 
合地理信息系统(GIS)等方法科学合理地布设观测 样带, 可提高观测结果的准确性; 生态系统演替动 态过程观测是生态阈值量化研究的重要手段, 也是 目前常用的一种野外数据获取方法(Li et al., 2014)。 大尺度的观测网络也是生态阈值研究的重要数据来 源, 可为各类模型提供校验数据, 解决大尺度的生 态学与环境相关问题(Mace, 2013)。很多国家都建立 了生态系统观测研究网络, 如美国国家生态观测网 络(The National Ecological Observatory Network, NEON)、澳大利亚陆地生态系统研究网络 (Terrestrial Ecosystem Research Network, TERN)、中 国生态系统研究网络(Chinese Ecosystem Research Network, CERN) (傅伯杰和刘宇, 2014)。大尺度生态 系统观测研究网络的建立, 可为准确检测和量化区 域尺度的生态阈值提供重要的数据来源(Gao et al., 2014)。

\section{2 统计分析方法}

基于生态阈值是生态系统非线性变化的拐点这 一重要认识, 采用统计学方法可量化生态阈值 (Toms \& Lesperance, 2003; Smith \& Tran, 2010; 吴 东浩等, 2010), 此方法是建立在获取数据的基础上 对实验数值进行描述, 产生简单的符合生态学规律 的统计模型(Qian \& Cuffney, 2012; Qian, 2014), 通 过统计模型确立符合环境标准的生态阈值。如通过 阶跃函数模型(step function model)来模拟生态阈值 发生的突变现象。Li等(2014)结合不同演替阶段生 态系统结构功能的野外观测, 利用非度量多维尺度 (nMDS)、分层分类分析 (hierarchical classification analysis)和分类回归树(C \& RT)方法量化评估中国 东部三江源退化高寒草甸(黑土滩)不同指标退化阈 值。Ruppert等(2012)运用Meta分析, 研究了干旱区 降水梯度上, 水分利用效率与地上生物量的阈值效 应。只有数据获取背景的一致性较高, 才能用Meta 分析法确定有应用价值的生态阈值, 目前Meta分析 的应用软件有Comprehensive Meta-analysis (CMA) 2.0、RevMan 5.0、Stata 10.0等(Borenstein et al., 2011)。

许多统计模型可以用来检测和量化生态阈值 (Qian, 2014), 但是因为很多研究需要通过模拟得出 变化点, 之后再找出该变化点代表的统计学意义, 应用存在一定的限制; 同时, 这类研究方法在一定 的程度上忽略了生态学的过程机理以及相互作用机
制。有研究将回归模型和生态足迹研究方法结合, 对景观进行聚类, 结合生态安全指数计算生态安全 阈值(张艳芳和任志远, 2006), 是统计学方法在生态 阈值计算中的新应用。

\section{3 模型模拟}

模型模拟较统计学方法能够实现更大时空尺度 阈值的确定与预测, 特别是可以充分发挥生态阈值 对地球上各种生态系统及其生态环境的预警作用。 可以利用模型模拟开展激发生态系统状态改变的驱 动力研究, 政策与管理方式变化等人类活动因素对 生态系统的影响等。目前我们可以用机理模型与系 统动力学模型来检测和量化生态阈值。

机理模型主要有生物地球化学模型与全球植被 动态模型。生物地球化学模型基于生态系统生产力 和水分平衡的过程机理。以气候、土壤条件和植被 类型为输入变量, 模拟环境因子(包括温度、降水和 辐射等)的变化对陆地生态系统碳水和养分循环的 影响, 主要有CENTURY模型、BIOME-BGC模型、 $\mathrm{TEM}$ 模型等。可以用于模拟单个或多个生态因子的 变化如何影响生态系统生产力和碳分配等方面的阈 值。全球植被动态模型可以揭示全球变化对生态系 统结构和功能两方面的影响, 主要有BIOME3模型、 Hybrid v 3.0模型和IBIS模型等, 可以模拟大范围的 植被动态等生态过程的阈值(Cramer et al., 1999, 2001)。例如, Wang等(2008)运用CENTURY模型研究 羊草(Leymus chinensis)草地放牧强度阈值。机理模 型较之统计模型能更好地反映生态学过程, 但是在 研究过程中, 由于数据不易获取, 只得将一些参数 设置为常数, 影响了结果的精确性。

系统动力学模型主要应用在生态学与社会经济 的交叉学科, 关系到地区综合与可持续发展。目前 在全球尺度上应用比较成熟的模型有Threshold 21, 用于国家社会、环境的可持续发展评估，帮助政策 制定者和决策者进行经济分析(Barney, 2002)。系统 动力学模型能够实现生态安全的动态阈值非线性变 化的模拟, 结合区域复合生态系统的特点, 建立由 经济、人口、资源、环境和生态 5 个子系统 71 个参数 构成的上海崇明岛区域生态安全的系统动力学模 型, 确定了生态安全的指标阈值, 同时认为阈值不 仅反映系统动态发展趋势, 而且是一定发展模式下 生态安全的特征参照系(李华和蔡永立, 2010); 选取 国内生产总值(GDP)、人口总量、环境污染量作为 
主要变量, 分别代表经济子系统、人口子系统和环 境子系统, 以粮食产出率、森林覆盖率、水土流失 率等指标作为其重要的参数因子, 选取水土流失面 积、净GDP、污染处理量等作为评价因子, 建立了 重庆三峡库区生态安全系统动力学模型, 在可持续 发展型、资源衰竭型和自然状态型3种情景下模拟确 定了生态安全的指标阈值(张梦婕等, 2015)。系统动 力学模型的优点是方便改动参数, 能够模拟较大时 间尺度的生态与社会经济复合过程, 模型的难点在 于系统因果反馈关系的建立, 以及涉及指标体系的 选取。

\section{3 生态阈值在生态学几个热点研究领域的 应用及未来研究展望}

\section{1 生态阈值的应用}

在任何系统动态中, 阈值都必不可少; 特别是 在可持续科学领域, 阈值研究是一个优先主题, 要 求大量基于试验和观测的数据来支持描述的稳态转 换(Walker \& Meyers, 2004)。生态阈值对生态系统结 构与功能的研究有着重要意义, 单个生态因子间阈 值的差异以及体现生态系统不同功能或者整体功能 的复合生态因子的关键國值, 都决定了生态阈值的 研究因不同尺度、不同层次, 不同研究目标而呈现 其特殊性。

\subsection{1 生态系统服务的阈值确定}

生态系统服务指人类从生态系统获得的各种惠 益, 千年生态系统评估关注不同生态系统的差异、 弹性与阈值 (Millennium Ecosystem Assessment, 2005)。生态系统服务中, 阈值研究的目的在于确定 人类对自然需求与生态系统调节功能之间的权衡关 系。生态系统服务具有复杂的尺度效应, 因此在阈 值确定过程中, 需要明确生态过程变化的机理, 并 且分析其驱动因素。目前的研究中, 主要是对生态 系统服务的价值量或物质量进行评估(傅伯杰和张 立伟, 2014), 而对生态系统服务阈值的关注比较 少。

\subsection{2 生物多样性保护阈值的确定}

生态阈值可以用来提高生物多样性保护和自然 资源管理成效(Huggett, 2005)。生物多样性是生态系 统结构和功能的基础, 关系到生态系统健康与人类 福祉。生境面积大小、生境破碎化、物种分布格局、 物种数量等因素都能引起生物多样性的改变。种的
分布和丰富度都受时空变化的影响。景观尺度发生 改变, 会通过很多环境与植被相互影响的过程, 激 发植物产生不同的响应, 不同种对景观尺度变化的 响应是不同的(Huggett, 2005)。当生境所在面积突破 一个给定阈值, 大量物种将从景观中消失, 因为现 存的每个相对小的种群都有着可以相互比较的灭绝 敏感性(Lindenmayer \& Luck, 2005), 因此保护区的 面积就是一个关键的阈值, 而这个阈值的确定就要 结合保护对象的不同而设置。动物要考虑其栖息地 和繁殖地, 植物要考虑传粉距离等。Banks-Leite等 (2014)评估了在生物多样性保护热点地区施行休耕 的利弊, 其研究认为政府对休耕土地提供生态补偿 可以提供更多的土地用于生物多样性保护，但如何 平衡经济支出与生态收益, 就需要应用生态阈值的 研究方法确定最适合的休耕面积。

\subsection{3 生态系统管理阈值的确定}

过去30年中, 生态学家与经济学家将生态阈值 概念应用到自然与人工生态系统的监测中, 主要用 作自然资源的保护与可持续性管理的主要概念基 础, 将生态阈值概念应用到保护濒危物种、模拟生 境退化及发展自然资源生产力的政策与法规等 (Huggett, 2005)。生态系统管理有别于传统的自然资 源管理, 把人类作为系统的一个组分, 对公众的特 性和需要更敏感, 它依赖模型和GIS等现代工具, 目标是区域长期的可持续发展(赵云龙等, 2004)。生 态阈值的研究结果是为管理服务的, 其概念已经应 用到了自然资源管理与政策制定方面, 如美国公共 土地管理政策。将阈值应用到管理中, 首先要对自 然生态系统进行分类, 对现有的状态进行评估, 如 果分类和评估出现问题, 错误地估计了管理对象的 状态, 之后的管理措施就会有偏差, 而且恢复工程 的费用将会很高(Briske et al., 2005), 如同医生给人 看病, 首先要诊断, 中医望闻问切, 西医开一堆化 验单, 之后才能对症下药。这个“症”的诊断就要用 到生态阈值。

\section{2 生态阈值研究展望}

\subsection{1 开展针对生态阈值的定量化研究}

生态阈值是生态系统对外界环境变化响应产生 状态改变的条件, 或者说是生态系统抵御外界干扰 的极限值或者耐受范围。而生态阈值产生的触发因 子不固定, 小的变化可能引发生态系统大的连锁反 应; 触发因子和生态系统响应发生在不同的时间维 
度, 我们发现隐藏在生态阈值与时差之下的重要生 态系统功能与过程, 对生态系统的研究非常必要。 目前对阈值的研究, 多是建立在单一的变化情景下, 如定义种的敏感性, 以及单一的环境因子梯度下的 研究, 对于多样性缺失、生物入侵、多世代稳定性 以及生境破碎化等因素共同作用的环境中, 需要发 展适合的定量化方法, 特别是针对复杂生态系统不 同过程的生态阈值量化方法。

\subsection{2 关注生态阈值的尺度效应并加强野外观测}

生态阈值具有复杂的时间与空间尺度效应。同 一对象在群落、区域和全球尺度上, 表现出不同的 生态阈值。确定大尺度上的生态阈值时应当考虑小 尺度的特异性变化。大尺度上发生稳态转换的突变 点, 可能就是源于小尺度上的量变积累; 如千里大 坝毁于蚁穴。又如在珍稀濒危植物保护中, 除了物 种本身生长过程中涉及的一些重要生理生态指标阈 值, 更重要的是其生境中的主要限制因子阈值以及 划定保护区的范围等这些大尺度的生态阈值。应当 加强不同尺度的野外观测, 为统计分析与模型模拟 提供有力和可信的数据支持。

\subsection{3 生态阈值在生态系统管理和“生态红线”划定 中的预警作用}

生态阈值研究的一个重要目的就是服务于管理 和决策。在生态系统管理中, 可以利用阈值的概念 对生态系统发展的各阶段进行更好的评估, 提高管 理和决策行为的可预测性。现有的阈值研究, 大多 针对生态退化阈值, 对于生态系统管理而言, 更有 效的阈值应当是预防國值, 在系统出现重大变化 (质变)之前, 预测并采取有效的管理手段, 干预退 化进程。

国家在生态环境敏感区、脆弱区划定“生态红 线”, 并针对各类功能区制定配套环境政策与标准, 保障国家和区域生态安全, 提高生态服务功能。我 们认为生态阈值研究是生态红线划定的数据基础和 科学依据。应用层面是生态红线, 背后的科学支撑 是生态阈值。生态红线相当于生态阈值概念中的红 色阈值, 是生态系统从量变到质变的关键点。在各 地操作层面上, 也可以适当降低生态红线等级, 以 黄色和橙色阈值作为生态红线的补充, 划分不同等 级的生态阈值供管理者参考, 便于地方经济发展和 生态环境保护的协调。

\section{基金项目 国家重点基础研究发展规划(973计划)}

课题(2014CB954303)。

\section{参考文献}

Armitage D, Béné C, Charles AT, Johnson D, Allison EH (2012). The interplay of well-being and resilience in applying a social-ecological perspective. Ecology and Society, 17, 15.

Bai YF, Wu JG, Clark CM, Naeem S, Pan QM, Huang JH, Zhang LX, Han XG (2010). Tradeoffs and thresholds in the effects of nitrogen addition on biodiversity and ecosystem functioning: Evidence from Inner Mongolia grasslands. Global Change Biology, 16, 358-372.

Banks-Leite C, Pardini R, Tambosi LR, Pearse WD, Bueno AA, Bruscagin RT, Condez TH, Dixo M, Igari AT, Martensen AC, Metzger JP (2014). Using ecological thresholds to evaluate the costs and benefits of set-asides in a biodiversity hotspot. Science, 345, 1041-1045.

Barney GO (2002). The Global 2000 Report to the President and the Threshold 21 model: Influences of Dana meadows and system dynamics. System Dynamics Review, 18, 123136.

Bestelmeyer BT (2006). Threshold concepts and their use in rangeland management and restoration: The good, the bad, and the insidious. Restoration Ecology, 14, 325-329.

Bestelmeyer BT, Herrick JE, Brown JR, Trujillo DA, Havstad KM (2004). Land management in the American southwest: A state-and-transition approach to ecosystem complexity. Environmental Management, 34, 38-51.

Borenstein M, Hedges LV, Higgins JPT, Rothstein HR (2011). Introduction to Meta-Analysis. 2nd edn. John Wiley \& Sons, New York.

Bowker MA, Miller ME, Garman SL, Belote T (2014). Applying threshold concepts to conservation management of dryland ecosystems: Case studies on the Colorado Plateau. In: Guntenspergen GR ed. Application of Threshold Concepts in Natural Resource Decision Making. Springer, New York. 101-130.

Briske DD, Fuhlendorf SD, Smeins FE (2005). State-andtransition models, thresholds, and rangeland health: A synthesis of ecological concepts and perspectives. Rangeland Ecology \& Management, 58, 1-10.

Cramer W, Bondeau A, Woodward FI, Prentice IC, Betts RA, Brovkin V, Cox PM, Fisher V, Foley JA, Friend AD, Kucharik C, Lomas MR, Ramankutty N, Sitch S, Smith B, White A, Young-Molling C (2001). Global response of terrestrial ecosystem structure and function to $\mathrm{CO}_{2}$ and climate change: Results from six dynamic global vegetation models. Global Change Biology, 7, 357-373.

Cramer W, Kicklighter DW, Bondeau A, Moore B III, Churkina G, Nemry B, Ruimy A, Schloss AL, The Participants of the Potsdam NPP Model Intercomparison (1999). Comparing global models of terrestrial net primary productivity (NPP): Overview and key results. Global 
Change Biology, 5, 1-15.

DeAngelis DL, Waterhouse JC (1987). Equilibrium and nonequilibrium concepts in ecological models. Ecological Monographs, 57, 1-21.

Ficetola GF, Denoël M (2009). Ecological thresholds: An assessment of methods to identify abrupt changes in species-habitat relationships. Ecography, 32, 1075-1084.

Folke C, Carpenter S, Walker B, Scheffer M, Elmqvist T, Gunderson L, Holling CS (2004). Regime shifts, resilience, and biodiversity in ecosystem management. Annual Review of Ecology, Evolution, and Systematics, 35, 557581.

Friedel MH (1991). Range condition assessment and the concept of thresholds: A viewpoint. Journal of Range Management, 44, 422-426.

Fu BJ, Liu Y (2014). Global ecosystem observation and research programs: Evolution and insights for future development. Progress in Geography, 33, 893-902. (in Chinese with English abstract) [傅伯杰, 刘宇 (2014). 国际生态 系统观测研究计划及启示. 地理科学进展, 33, 893902.]

Fu BJ, Zhang LW (2014). Land-use change and ecosystem services: Concepts, methods and progress. Progress in Geography, 33, 441-446. (in Chinese with English abstract) [傅伯杰, 张立伟. (2014). 土地利用变化与生态 系统服务: 概念、方法与进展. 地理科学进展, 33, 441-446.]

Future Earth (2013). Future Earth initial design: Report of the transition team. Paris, FRA: International Council for Science (ICSU). http://www.icsu.org/future-earth/mediacentre/relevant_publications. Cited: 2014-12-26.

Gao Y, Zhu XJ, Yu GR, He NP, Wang QF, Tian J (2014). Water use efficiency threshold for terrestrial ecosystem carbon sequestration in China under afforestation. Agricultural and Forest Meteorology, 195-196, 32-37.

Groffman PM, Baron JS, Blett T, Gold AJ, Goodman I, Gunderson LH, Levinson BM, Palmer MA, Paerl HW, Peterson GD, Poff NL, Rejeski DW, Reynolds JF, Turner MG, Weathers KC, Wiens J (2006). Ecological thresholds: The key to successful environmental management or an important concept with no practical application. Ecosystems, 9, 1-13.

Holling CS (1973). Resilience and stability of ecological systems. Annual Review of Ecology and Systematics, 4, 1-23.

Huggett AJ (2005). The concept and utility of "ecological thresholds" in biodiversity conservation. Biological Conservation, 124, 301-310.

Kates RW, Clark WC, Corell R, Hall JM, Jaeger CC, Lowe I, McCarthy JJ, Schellnhuber HJ, Bolin B, Dickson NM, Faucheux S, Gallopin GC, Grübler A, Huntley B, Jäger J, Jodha NS, Kasperson RE, Mabogunje A, Matson P, Mooney H, Moore B III, O’Riordan T, Svedin U (2001).
Environment and development: Sustainability science. Science, 292, 641-642.

Larsen S, Alp M (2015). Ecological thresholds and riparian wetlands: An overview for environmental managers. Limnology, 16, 1-9.

Laycock WA (1991). Stable states and thresholds of range condition on north American rangelands: A viewpoint. Journal of Range Management, 44, 427-433.

Li H, Cai YL (2010). The threshold of eco-security based on system dynamics and application: A case study of Chongming Island. Acta Ecologica Sinica, 30, 3654-3664. (in Chinese with English abstract) [李华, 蔡永立 (2010). 基 于SD的生态安全指标阈值的确定及应用——上海崇 明岛为例. 生态学报, 30, 3654-3664.]

Li XL, Perry GLW, Brierley G, Sun HQ, Li CH, Lu GX (2014). Quantitative assessment of degradation classifications for degraded alpine meadows (Heitutan), Sanjiangyuan, Western China. Land Degradation \& Development, 25, 417-427.

Lindenmayer DB, Luck G (2005). Synthesis: Thresholds in conservation and management. Biological Conservation, 124, 351-354.

Mace G (2013). Global change: Ecology must evolve. Nature, 503, 191-192.

Martin J, Runge MC, Nichols JD, Lubow BC, Kendall WL (2009). Structured decision making as a conceptual framework to identify thresholds for conservation and management. Ecological Applications, 19, 1079-1090.

May RM (1973). Stability and Complexity in Model Ecosystems. Princeton University Press, Princeton, USA.

May RM (1977). Thresholds and breakpoints in ecosystems with a multiplicity of stable states. Nature, 269, 471-477.

Meyers J, Walker BH (2003). Thresholds and alternate states in ecological and social-ecological systems: Thresholds database. Resilience Alliance. http://www.resalliance.org. au. Cited: 2014-12-26.

Millennium Ecosystem Assessment (2005). Ecosystems and Human Well-Being. 2nd edn. Island Press, Washington, DC.

Muradian R (2001). Ecological thresholds: A survey. Ecological Economics, 38, 7-24.

Perrings C, Walker B (1997). Biodiversity, resilience and the control of ecological-economic systems: The case of fire-driven rangelands. Ecological Economics, 22, 73-83.

Qian SS (2014). Ecological threshold and environmental management: A note on statistical methods for detecting thresholds. Ecological Indicators, 38, 192-197.

Qian SS, Cuffney TF (2012). To threshold or not to threshold? That's the question. Ecological Indicators, 15, 1-9.

Rial JA, Pielke RA, Beniston M, Claussen M, Canadell J, Cox P, Held H, de Noblet-Ducoudré N, Prinn R, Reynolds JF, Salas JD (2004). Nonlinearities, feedbacks and critical 
thresholds within the Earth's climate system. Climatic Change, 65, 11-38.

Ruppert JC, Holm A, Miehe S, Muldavin E, Snyman HA, Wesche K, Linstadter A (2012). Meta-analysis of ANPP and rain-use efficiency confirms indicative value for degradation and supports non-linear response along precipitation gradients in drylands. Journal of Vegetation Science, 23, 1035-1050.

Sasaki T, Okayasu T, Jamsran U, Takeuchi K (2008). Threshold changes in vegetation along a grazing gradient in Mongolian rangelands. Journal of Ecology, 96, 145-154.

Scheffer M, Bascompte J, Brock WA, Brovkin V, Carpenter SR, Dakos V, Held H, van Nes EH, Rietkerk M, Sugihara G (2009). Early-warning signals for critical transitions. Nature, 461, 53-59.

Scheffer M, Carpenter SR, Lenton TM, Bascompte J, Brock W, Dakos V, van de Koppel J, van de Leemput IA, Levin SA, van Nes EH, Pascual M, Vandermeer J (2012). Anticipating critical transitions. Science, 338, 344-348.

Smith AJ, Tran CP (2010). A weight-of-evidence approach to define nutrient criteria protective of aquatic life in large rivers. Journal of the North American Benthological Society, 29, 875-891.

Suding KN, Gross KL, Houseman GR (2004). Alternative states and positive feedbacks in restoration ecology. Trends in Ecology \& Evolution, 19, 46-53.

Suding KN, Hobbs RJ (2009). Threshold models in restoration and conservation: A developing framework. Trends in Ecology \& Evolution, 24, 271-279.

Swift TL, Hannon SJ (2010). Critical thresholds associated with habitat loss: A review of the concepts, evidence, and applications. Biological Reviews, 85, 35-53.

Toms JD, Lesperance ML (2003). Piecewise regression: A tool for identifying ecological thresholds. Ecology, 84, 2034-2041.

Twidwell D, Fuhlendorf SD, Taylor CA, Rogers WE (2013). Refining thresholds in coupled fire-vegetation models to improve management of encroaching woody plants in grasslands. Journal of Applied Ecology, 50, 603-613.

Walker B, Meyers JA (2004). Thresholds in ecological and social-ecological systems: A developing database. Ecology and Society, 9, 3.

Wang C, Wang X, Liu D, Wu H, Lü X, Fang Y, Cheng W, Luo W, Jiang P, Shi J, Yin H, Zhou J, Han X, Bai E (2014).
Aridity threshold in controlling ecosystem nitrogen cycling in arid and semi-arid grasslands. Nature Communications, 5, 4799.

Wang YH, Zhou GS, Jia BR (2008). Modeling SOC and NPP responses of meadow steppe to different grazing intensities in northeast China. Ecological Modelling, 217, 72-78.

Wu DH, Yu HY, Wu HY, Zhou B, Wang BX (2010). Estimation of river nutrients thresholds based on benthic macroinvertebrate assemblages: A case study in the upper reaches of Xitiao Stream in Zhejiang, China. Chinese Journal of Applied Ecology, 21, 483-488. (in Chinese with English abstract) [吴东浩, 于海燕, 吴海燕, 周斌, 王备 新 (2010). 基于大型底栖无脊椎动物确定河流营养盐 浓度國值一以西苕溪上游流域为例. 应用生态学报, 2, 483-488.]

Zhang MJ, Guan DJ, Su WC (2015). Scenarios simulation and indices thresholds determination of ecological security in three gorges reservoir based on system dynamics. Acta Ecologica Sinica, 35, 4880-4890. (in Chinese with English abstract) [张梦婕, 官冬杰, 苏维词 (2015). 基于系 统动力学的重庆三峡库区生态安全情景模拟及指标阈 值确定. 生态学报, 35, 4880-4890.]

Zhang YF, Ren ZY (2006). Discussion of the ways to quantificational evaluation and threshold value of regional ecological security. Journal of Arid Land Resources and Environment, 20(2), 11-16. (in Chinese with English abstract) [张艳芳, 任志远 (2006). 区域生态安全定量 评价与阈值确定的方法探讨. 干旱区资源与环境, 20(2), 11-16.]

Zhao HX, Wu SH, Jiang LG (2007). Review on recent advances in ecological threshold research. Acta Ecologica Sinica, 27, 338-345. (in Chinese with English abstract) [赵 慧霞, 吴绍洪, 姜鲁光 (2007). 生态阈值研究进展. 生 态学报, 27, 338-345.]

Zhao YL, Tang HP, Chen H, Qin XY, Li XY (2004). Connotation and application of ecosystem management. Geography and Geo-Information Science, 20(6), 94-98. (in Chinese with English abstract) [赵云龙, 唐海萍, 陈 海, 秦向阳, 李新宇 (2004). 生态系统管理的内涵与应 用. 地理与地理信息科学, 20(6), 94-98.]

责任编委：彭少麟 责任编辑：王 葳 